\title{
Hypochondriac Region
}

National Cancer Institute

\section{Source}

National Cancer Institute. Hypochondriac Region. NCI Thesaurus. Code C34003.

The superior portion of the abdomen that lies inferior to the right and left costal marg ins and lateral to the epigastrum on both the right and left sides of the body. 